\title{
Cystoid Macular Edema Associated with Oral Antineoplastic Agent S-1 in a Patient with Diabetic Retinopathy
}

\author{
Tomomi Higashide Eiji Murotani Kazuhisa Sugiyama \\ Department of Ophthalmology and Visual Science, Kanazawa University \\ Graduate School of Medical Science, Kanazawa, Japan
}

\section{Key Words}

Anemia - Bevacizumab - Cystoid macular edema - Diabetic retinopathy Gastric cancer $\cdot$ Neovascular glaucoma $\cdot$ S-1

\begin{abstract}
A 60-year-old man with neovascular glaucoma due to diabetic retinopathy received an intravitreal injection of $1.25 \mathrm{mg}$ bevacizumab (IVB) followed by extensive panretinal photocoagulation in the right eye. The anterior segment neovascularization regressed within 10 days after IVB. One and a half months later, the patient underwent gastrectomy for stage IIIb gastric cancer. Two months later, he was started on S- 1 orally $(100 \mathrm{mg} /$ day for 48,26 , and 32 consecutive days in the first, second, and third treatment cycle, respectively). The interval between the first and second treatment cycle was 20 days and between the second and third cycle it was 24 days. The patient developed anemia and diarrhea. At the end of the second S-1 cycle, cystoid macular edema developed in the right eye, although diabetic retinopathy and neovascular glaucoma were stable. Macular edema persisted for 5 months despite another IVB, and disappeared 3 months after termination of S-1 therapy. The time course of the magnitude of macular edema correlated well with the severity of anemia. The macular edema was possibly associated with anemia, which is a major side effect of S-1. Further studies are warranted to investigate the relationship between anemia and macular edema in patients with diabetic retinopathy.
\end{abstract}




\section{Introduction}

S-1 is an oral antineoplastic agent consisting of the fluorouracil (5-FU) prodrug tegafur combined with two modulators, 5-chloro-2,4-dihydroxypyridine and potassium oxonate. S-1-based chemotherapy is the standard treatment of advanced gastric cancer in Japan [1]. Although S-1 is designed to be more active and less toxic than intravenous 5-FU with fewer gastrointestinal side effects, ocular surface and lacrimal complications have been reported [2,3]. In contrast, retinal adverse events were not found through a literature search. We present a case with diabetic retinopathy and neovascular glaucoma that developed cystoid macular edema during postoperative adjunctive chemotherapy of S-1 for advanced gastric cancer. The macular edema was possibly associated with anemia, a major side effect of S-1 [4].

\section{Case Presentation}

A 60-year-old man was referred to our department for the treatment of neovascular glaucoma due to diabetic retinopathy in the right eye. Visual acuity was 0.4 in the right eye, and there was no light perception in the left eye. Intraocular pressures were 12 and $18 \mathrm{~mm} \mathrm{Hg}$ in the right and left eyes, respectively, under maximum antiglaucoma medications. Slit-lamp examination disclosed marked iris rubeosis, a mostly open angle with prominent neovascularization in the right eye, a scarred bleb after trabeculectomy and less active anterior segment neovascularization in the left eye, and nuclear cataract in both eyes. Fundus examination revealed scattered blot hemorrhages, cotton wool spots and scattered laser scars (fig. 1a). Glaucomatous optic disc cupping was present in both eyes, and the optic disc in the left eye was totally pale. Macular edema which was examined by optical coherence tomography (OCT; Stratus OCT, Carl Zeiss Meditec, Dublin, Calif., USA) was absent in both eyes (fig. $1 b, c)$.

An intravitreal injection of $1.25 \mathrm{mg}$ bevacizumab (IVB), an antibody against vascular endothelial growth factor (VEGF), followed by extensive panretinal photocoagulation was performed in the right eye, which resulted in regression of anterior segment neovascularization within 10 days after IVB. Off-label use of bevacizumab was approved by the Institutional Review Board of the Kanazawa University Graduate School of Medical Science, Kanazawa, Japan. Informed consent was obtained from the patient regarding treatment of the right eye after thorough discussion, especially concerning bevacizumab. One and a half months later, he underwent gastrectomy for stage IIIb gastric cancer. Two months after gastrectomy, the patient underwent phacoemulsification and intraocular lens implantation in the right eye, with subsequent improvement in vision to 0.9. Prior to cataract surgery, a second IVB was performed to prevent postoperative macular edema. Nine days after cataract surgery, he was started on S-1 orally $(100 \mathrm{mg}$ /day for 48, 26, 32 consecutive days in the first, second, and third treatment cycle, respectively). The interval between the first and second treatment cycle was 20 days, and between the second and third cycle it was 24 days.

At the end of the second cycle, 3 months after cataract surgery, vision in his right eye decreased to 0.4 . Emergence of cystoid macular edema in the right eye was confirmed by OCT (fig. $1 \mathrm{~d}-\mathrm{f}$ ). Since we could observe the edema clearly in the OCT images, and diabetic retinopathy and neovascular glaucoma were stable, we did not perform fluorescein angiography as a follow-up examination of the edema. Diffuse superficial keratitis developed in the right eye, which was successfully treated using a disposable soft contact lens and sodium hyaluronate eye drops. Macular edema persisted for 5 months despite another IVB (fig. 1g-i). Macular edema disappeared 3 months after termination of S-1 therapy (fig. 1j-l). Vision in the right eye returned to 0.9 . The time course of the 1 -mm central retinal thickness correlated well with that of the severity of anemia ( $\underline{\text { fig. } 2}$ ). No recurrence of macular edema was observed for more than 1 year. 


\section{Discussion}

Cystoid macular edema developed in a patient with diabetic retinopathy and neovascular glaucoma. Macular edema is a common complication of diabetic retinopathy and VEGF is believed to contribute to its pathogenesis. Highly elevated intraocular concentration of VEGF was reported in eyes with neovascular glaucoma [5]. This patient received panretinal photocoagulation and cataract surgery, each of which may have induced macular edema. IVB was recently reported to suppress macular edema associated with these interventions in patients with diabetic retinopathy [6,7]. In this case, bevacizumab was injected prior to both therapies. Given that macular edema emerged 6 and 3 months after initiation of panretinal photocoagulation and cataract surgery, respectively, these procedures were unlikely a primary initiator of macular edema. Fundus examination also excluded the presence of other retinopathies that can cause macular edema, such as retinal vein occlusion. Worsening of diabetic retinopathy and neovascular glaucoma did not occur during the follow-up period. Thus, no intraocular findings could explain the emergence of macular edema.

The patient underwent gastrectomy, and macular edema occurred during the period of postoperative S-1 chemotherapy. The patient also developed diffuse superficial keratitis. The ocular surface damage by S-1 is thought to be a direct toxicity of the drug in the tear film [3]. However, no studies, including a clinical trial of intravitreal injections, have shown any 5-FU-induced morphological alterations in the retina [8]. Therefore, macular edema was unlikely to be caused by a direct toxicity of 5-FU.

Systemic side effects of S-1 include myelosuppression, gastrointestinal toxicity, and skin reactions. This patient developed anemia and diarrhea during S-1 treatment. Hemoglobin concentration decreased below $10 \mathrm{~g} / \mathrm{dl}$, which corresponds to grade 2 anemia, according to the National Cancer Institute Common Toxicity Criteria for Adverse Events (version 2.0). The incidence of grade 2 or more anemia was $33 \%$ in patients using S-1 in a large-scale trial of postoperative adjuvant chemotherapy for advanced gastric cancer conducted in Japan [4]. It is noteworthy that the time course of the severity of anemia correlated well with that of the magnitude of macular edema. It is plausible that retinal hypoxia due to diabetic retinopathy became worse as a consequence of S-1-induced anemia, which resulted in the emergence of macular edema.

Regarding drug-induced macular edema in patients with diabetes, thiazolidinediones, a class of drug that binds the peroxisome proliferator-activated receptor- $\gamma$ to reduce insulin resistance in type 2 diabetes, have been reported to be associated with macular edema [9]. This patient had no history of taking this drug. Macular edema is thought to be caused by drug-induced systemic fluid retention [9]. However, mild anemia is also a major side effect of thiazolidinediones [10]. Further studies are warranted to investigate the relationship between anemia and macular edema in patients with diabetes.

\section{Disclosure Statement}

The authors declare that they have no conflicts of interest. 

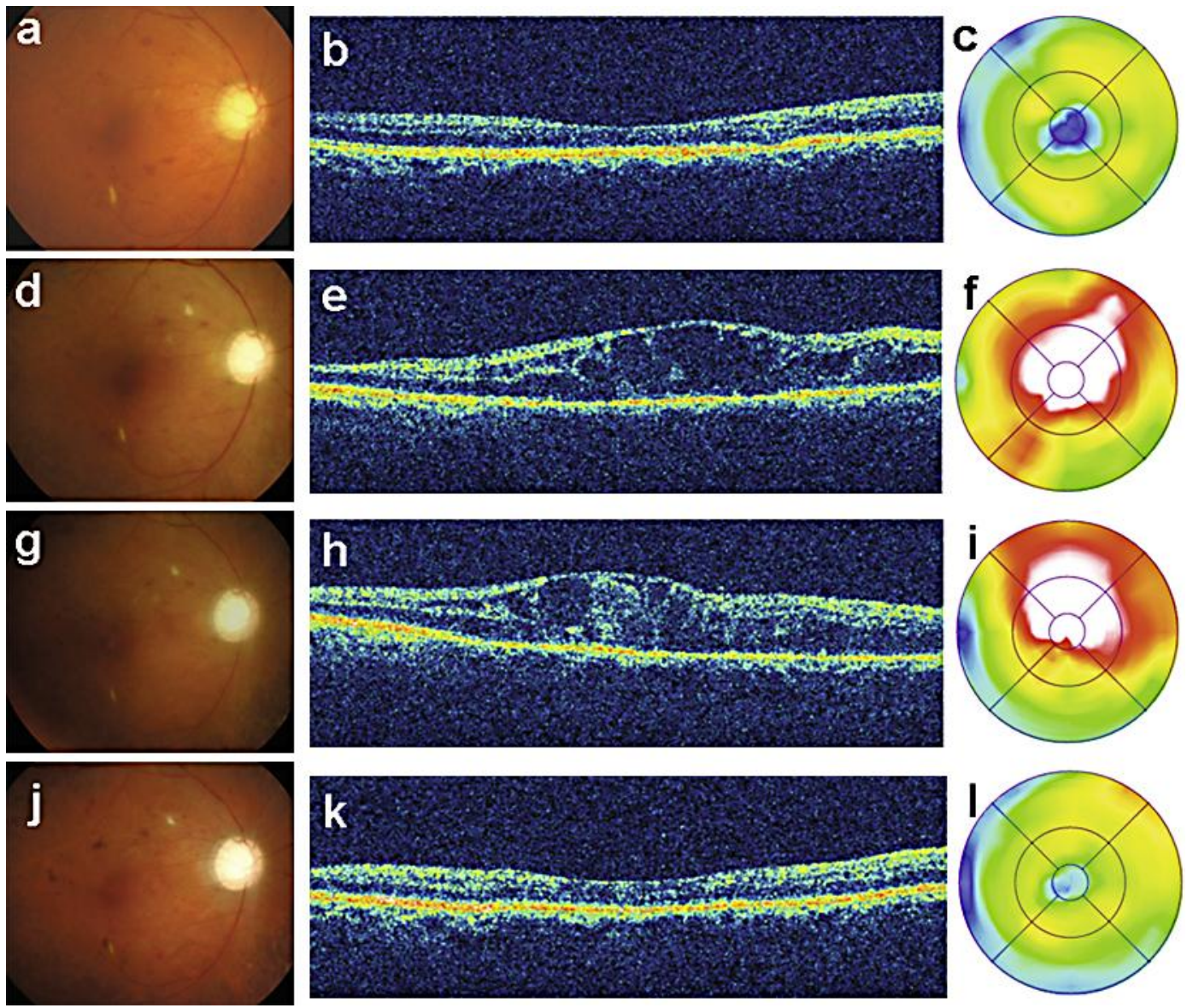

Fig. 1. Fundus photographs and OCT images of the right eye. a-c Macular edema was absent at the first visit to our department. $\mathbf{d}-\mathbf{f}$ Cystoid macular edema emerged at the end of the second S- 1 cycle. $\mathbf{g}-\mathbf{i}$ Macular edema persisted 1 month after an intravitreal injection of $1.25 \mathrm{mg}$ bevacizumab. $\mathrm{j}-\mathrm{I}$ Macular edema disappeared 3 months after termination of $S-1$ therapy. $\mathbf{a}, \mathbf{d}, \mathbf{g}, \mathbf{j}$ Fundus photographs. $\mathbf{b}, \mathbf{e}, \mathbf{h}, \mathbf{k}$ Horizontal line scans through the fovea. c, f, i, I Macular thickness maps. 


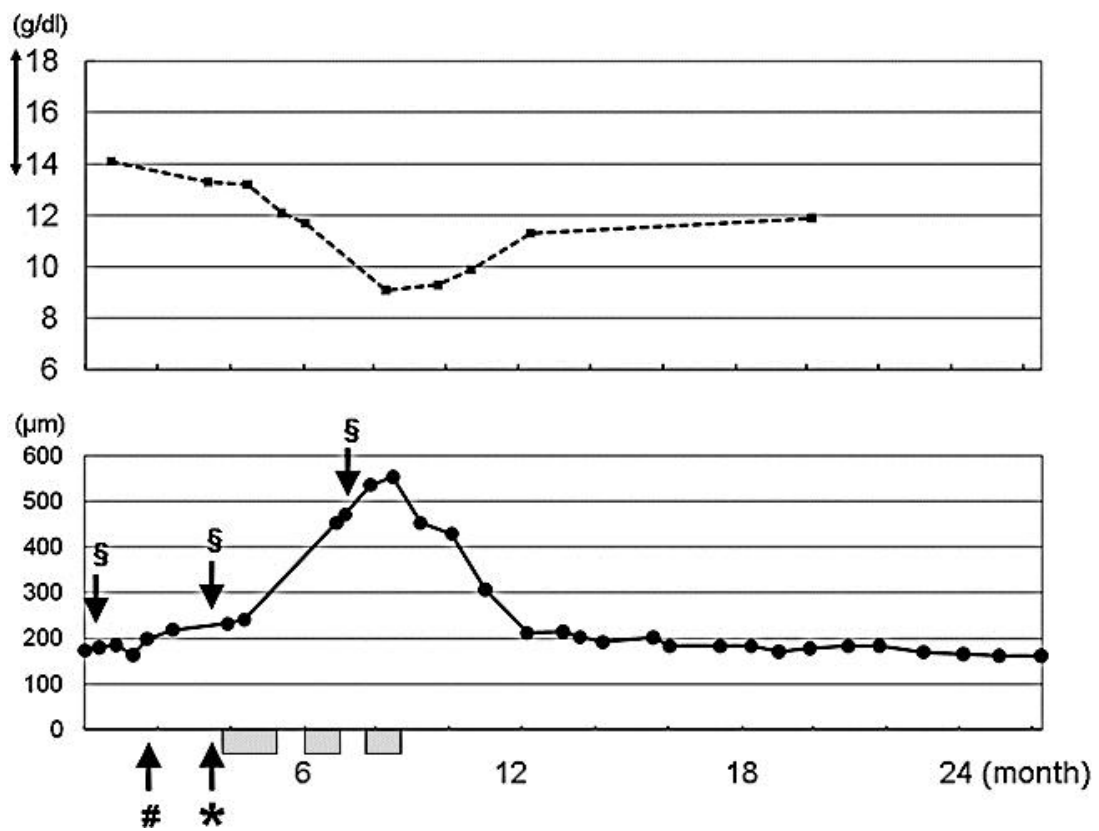

Fig. 2. Relationship between the time course of anemia and macular edema in the right eye. Upper graph: hemoglobin concentration. The vertical double-headed arrow indicates the normal range. Lower graph: the 1-mm central retinal thickness in OCT images. \# Gastrectomy. * Cataract surgery. $\S$ Intravitreal injections of $1.25 \mathrm{mg}$ bevacizumab. Boxes in the horizontal axis indicate the periods of S-1 treatment. Horizontal axes of both graphs indicate months after the first visit to our department.

\section{References}

1 Kubota T: The role of S-1 in the treatment of gastric cancer. Br J Cancer 2008;98:1301-1304.

-2 Esmaeli B, Golio D, Lubecki L, Ajani: Canalicular and nasolacrimal duct blockage: an ocular side effect associated with the antineoplastic drug S-1. Am J Ophthalmol 2005;140:325-327.

-3 Chikama T, Takahashi N, Wakuta M, Nishida T: Noninvasive direct detection of ocular mucositis by in vivo confocal microscopy in patients treated with S-1. Mol Vis 2009;15:2896-2904.

-4 Sakuramoto S, Sasako M, Yamaguchi T, Kinoshita T, Fujii M, Nashimoto A, Furukawa H, Nakajima T, Ohashi Y, Imamura H, Higashino M, Yamamura Y, Kurita A, Arai K; ACTS-GC Group: Adjuvant chemotherapy for gastric cancer with S-1, an oral fluoropyrimidine. N Engl J Med 2007;357:1810-1820.

$\checkmark 5$ Tripathi RC, Li J, Tripathi BJ, Chalam KV, Adamis AP: Increased level of vascular endothelial growth factor in aqueous humor of patients with neovascular glaucoma. Ophthalmology 1998;105:232-237.

-6 Mason J0 3rd, Yunker JJ, Vail R, McGwin G Jr: Intravitreal bevacizumab (Avastin) prevention of panretinal photocoagulation-induced complications in patients with severe proliferative diabetic retinopathy. Retina 2008;28:1319-1324.

-7 Cheema RA, Al-Mubarak MM, Amin YM, Cheema MA: Role of combined cataract surgery and intravitreal bevacizumab injection in preventing progression of diabetic retinopathy: prospective randomized study. J Cataract Refract Surg 2009;35:18-25.

-8 Blankenship GW: Evaluation of a single intravitreal injection of 5-fluorouracil in vitrectomy cases. Graefes Arch Clin Exp Ophthalmol 1989;227:565-568.

-9 Fong DS, Contreras R: Glitazone use associated with diabetic macular edema. Am J Ophthalmol 2009;147:583-586.e1.

10 Berria R, Glass L, Mahankali A, Miyazaki Y, Monroy A, De Filippis E, Cusi K, Cersosimo E, Defronzo RA, Gastaldelli A: Reduction in hematocrit and hemoglobin following pioglitazone treatment is not hemodilutional in type II diabetes mellitus. Clin Pharmacol Ther 2007;82:275-281. 